\title{
Phase 1 dose escalation of ONT-10, a therapeutic MUC1 vaccine, in patients with advanced cancer
}

\author{
John Nemunaitis ${ }^{1 *}$, Cynthia Bedell ${ }^{1}$, Kevin Klucher $^{2}$, Alex Vo², Sam Whiting ${ }^{2}$ \\ From Society for Immunotherapy of Cancer 28th Annual Meeting \\ National Harbor, MD, USA. 8-10 November 2013
}

\section{Background}

Mucin 1 (MUC1), a glycoprotein normally expressed at low levels on the apical borders of secretory epithelial cells, is overexpressed and aberrantly glycosylated in many cancers. ONT 10 is a therapeutic peptide vaccine incorporating a synthetic glycolipopeptide MUC1 antigen, M40Tn6, and novel synthetic TLR-4 agonist, PET Lipid A, in a liposomal formulation designed to elicit antibody and cellular immune responses.

\section{Methods}

A phase 1 study was initiated to evaluate the safety and tolerability of ONT-10, as well as cellular and humoral immune responses and antitumor activity. Patients (pts) with incurable solid tumors associated with MUC1 expression were eligible. Cyclophosphamide $250 \mathrm{mg} / \mathrm{m} 2$ IV was given on day -3 followed by ONT-10 at the cohort-specific dose $(250 \mu \mathrm{g}, 500 \mu \mathrm{g}$ or $1000 \mu \mathrm{g})$ subcutaneously day 1 and then Q2W for 4 total doses or QW for 8 total doses in a $3+3$ dose escalation design. Immune response was assessed by serum titers of MUC1-specific antibodies using M40Tn6 ELISA and by MUC1-specific ELISPOT for interferon gamma. Tumor response was assessed by RECIST 1.1 and immune-related response criteria (irRC). Pts without progressive disease by irRC were eligible for a maintenance protocol to receive ONT10 every 6 weeks.

\section{Results}

The study is ongoing and 28 pts have been treated. Diagnoses were: ovarian/primary peritoneal $(n=10)$, pancreatic $(n=5)$, colorectal $(n=3)$, endometrial $(n=3)$, breast $(n=2)$, lung, bladder, cervical, duodenal, and prostate ( $\mathrm{n}=1$ each). Median prior lines of therapy 4 (range 1 - 11); median age 61.5 years (range 35 - 77); all pts had ECOG status of 0/1.
No DLTs have occurred at doses up to $1000 \mu \mathrm{g}$ Q2W and $500 \mu \mathrm{g}$ QW. 90\% of AEs have been Grade 1-2; the most common ( $\geq 20 \%)$ being fatigue $(40 \%)$, abdominal pain $(28 \%)$, nausea $(28 \%)$ and constipation $(24 \%)$. The most common treatment-related AEs (TRAEs) have been fatigue (32\%) and injection site reactions (20\%) and all TRAEs have been Grade 1-2. MUC1 specific antibody responses were seen in the majority of pts. Cellular response assessment is ongoing. Best tumor response in 25 evaluable pts was SD (17 pts; $68 \%$ ) and PD (8 pts; 32\%). One pt with ovarian cancer had $16 \%$ tumor shrinkage. SD $\geq 6$ mo was seen in $28 \%$ of patients including ovarian/primary peritoneal $(n=3)$ and endometrial, breast, colon, pancreatic $(n=1$ each). 17 pts have enrolled on the maintenance study.

\section{Conclusions}

In a diverse population of late stage cancer pts, ONT-10 was well tolerated at up to $1000 \mu \mathrm{g}$ Q2W and $500 \mu \mathrm{g}$ QW. Prolonged disease control and encouraging immune responses have been seen. Updated results and immune data will be presented.

\section{Authors' details \\ ${ }^{1}$ Mary Crowley Cancer Research Center, Dallas, TX, USA. ${ }^{2}$ Oncothyreon, Inc., Seattle, WA, USA.}

Published: 7 November 2013

doi:10.1186/2051-1426-1-S1-P240

Cite this article as: Nemunaitis et al:: Phase 1 dose escalation of ONT-10, a therapeutic MUC1 vaccine, in patients with advanced cancer. Journal for ImmunoTherapy of Cancer 2013 1(Suppl 1):P240.

${ }^{1}$ Mary Crowley Cancer Research Center, Dallas, TX, USA

Full list of author information is available at the end of the article

(c) 2013 Nemunaitis et al; licensee BioMed Central Ltd. This is an Open Access article distributed under the terms of the Creative 